\title{
CONTROLLING THE INTERNAL MICROSTRUCTURE AND PROPERTIES OF PHYSICALLY CROSS-LINKED PVA HYDROGELS BY THE RATE OF FREEZING
}

\author{
${ }^{1}$ Monika TRUDICOVA, ${ }^{1}$ Jan ZAHRADKA, ${ }^{1}$ Petr SEDLACEK, ${ }^{1}$ Miloslav PEKAR \\ 'Brno university of technology, FCH, Brno, Czech Republic, xctrudicova@fch.vut.cz
}

https://doi.org/10.37904/nanocon.2020.3769

\begin{abstract}
PVA hydrogels can be formed via physical crosslinking (e.g. by cyclic freezing/thawing of aqueous polyvinyl alcohol solutions) or with a chemical cross-linker. Physical crosslinking is more useful for biomedical applications since it contains no harmful cross-linker residues. This work, therefore, focused on studying properties of physically crosslinked hydrogels with different concentrations of polyvinyl alcohol (2.5 to $15 \mathrm{wt} \%)$ prepared via cyclic freezing/thawing under different cryogenic conditions: in the liquid nitrogen $\left(-196{ }^{\circ} \mathrm{C}\right)$, in two different laboratory freezers $\left(-80^{\circ} \mathrm{C}\right.$ and $\left.-18^{\circ} \mathrm{C}\right)$ and the ice bath. Furthermore, the effects of the addition of several cryoprotectants were evaluated. The optical and mechanical properties of the prepared samples were studied, and the microstructure of the hydrogels was observed by scanning electron microscopy after freeze-drying.
\end{abstract}

Keywords: Hydrogel, polyvinyl alcohol, rheology, scanning electron microscopy

\section{INTRODUCTION}

Hydrogels are defined as systems consisting of a three-dimensional network of polymer chains and water filling the space between these macromolecules. They can be used in a wide range of medical applications, such as wound dressings, controlled drug release, or as scaffolds in tissue engineering [1]. Optical, mechanical, and transport properties of hydrogels are crucial for their potential applications; therefore, the possibility of modifying their properties and relevant material parameters according to the specific needs of a particular application is still being researched [2,3]. One of the basic tools for modifying the material properties of hydrogels is a manipulation of the internal chemical and morphological architecture [4].

This work focuses on the possibilities of modifying optical and mechanical properties of polyvinyl alcohol hydrogels via adjustment of their preparation procedure. It aims to understand the way how these properties can be controlled by manipulating the internal structure in physically crosslinked polyvinyl alcohol hydrogels.

\section{EXPERIMENTAL PART}

\subsection{Preparation of polyvinyl alcohol hydrogels}

Polyvinyl alcohol (PVA) is one of the synthetic polymers most widely used for the preparation of hydrogels for biomedical applications. It is a linear polymer, which is produced by radical polymerization of vinyl acetate and subsequent hydrolysis of acetate groups to hydroxyl groups, the degree of this hydrolysis (usually $85-100 \%$ ) determining the properties of the polymer such as solubility in aqueous solutions, etc. [5]. The PVA hydrogel can be formed either by physical crosslinking by repeated freezing cycles or by chemical crosslinking with crosslinkers [6]. In this work, all hydrogels were prepared via cyclic freezing/thawing. The polyvinyl alcohol used in this work was purchased from Sigma Aldrich, average Mw 130,000, 99+\% hydrolyzed; CAS number 9002-89-5. To monitor the effect of PVA content in the gel, hydrogels were prepared from aqueous solutions 
with different concentrations of polyvinyl alcohol (2.5, 5.0, 7.5, 10.0, 12.5 and $15.0 \mathrm{wt} \%$ ) by cyclic freezing/thawing in the freezer $\left(-18^{\circ} \mathrm{C}\right)$.

Furthermore, for the gels prepared from PVA solution with a concentration of $10.0 \mathrm{wt} \%$ of polyvinyl alcohol, additives in the form of cryoprotectants were added in concentrations of $10.0 \mathrm{wt} \%$ to prevent damage to the three-dimensional polymer network during freezing due to the restriction of ice crystal growth. Trehalose, sucrose, urea, and glycerol were used as cryoprotectants. These samples were also prepared in the freezer $\left(-18^{\circ} \mathrm{C}\right)$.

According to the literature, the properties of a polyvinyl alcohol hydrogel can be influenced both by the concentration of PVA and by the temperature of freezing that defines the freezing rate [7]. Therefore, in another set of experiments four different cryogenic conditions were tested: the liquid nitrogen $\left(-196{ }^{\circ} \mathrm{C}\right)$, two different laboratory freezers $\left(-80^{\circ} \mathrm{C}\right.$ and $\left.-18{ }^{\circ} \mathrm{C}\right)$, and the ice bath. For these experiments were prepared hydrogels with $10.0 \mathrm{wt} \%$ of polyvinyl alcohol. A series of hydrogels with different concentrations of polyvinyl alcohol (2.5, $5.0,7.5,10.0,12.5$, and $15 \mathrm{wt} \%$ ) were prepared in the ice bath for comparison with the reference samples prepared previously in the freezer $\left(-18^{\circ} \mathrm{C}\right)$.

\subsection{Study of optical properties}

The first proposed method for evaluating the optical properties of hydrogels was an objective photometric analysis of samples using a spectrophotometer. However, most of the samples did not have sufficient transmittance, therefore the optical properties were evaluated visually. The samples were placed on the pad with an auxiliary cross, which formed a reference point for comparing the transparency (Figure 1).

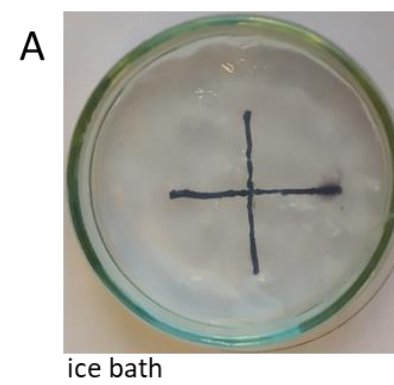

B

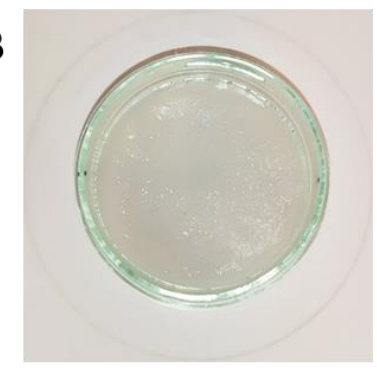

trehalose
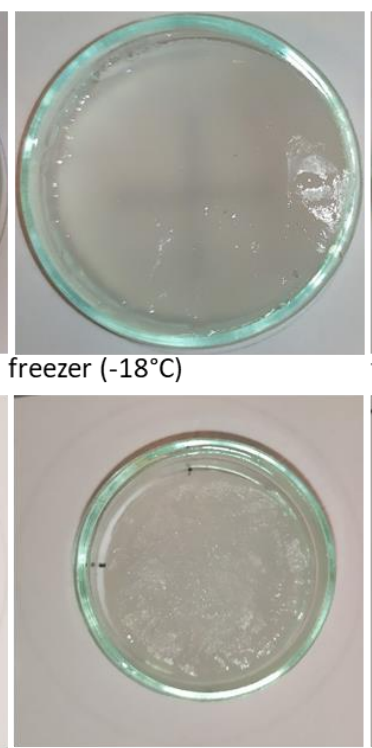

sucrose
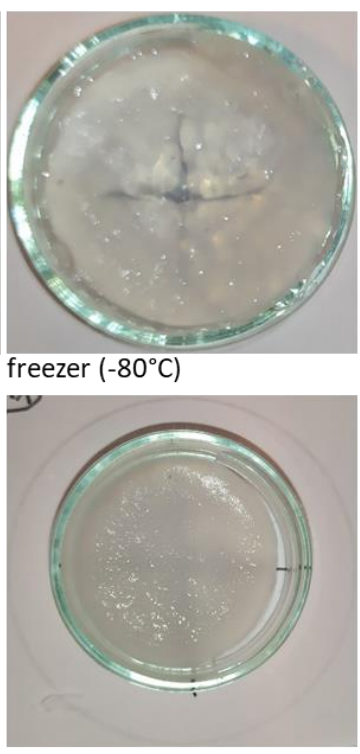

glycerol
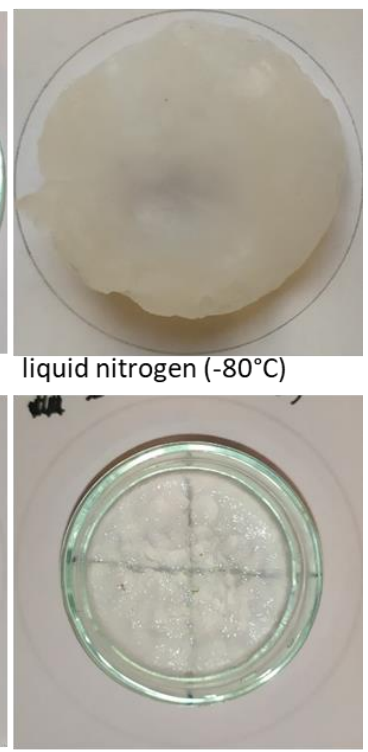

urea

Figure 1 Visual evaluation of samples with concentration $10.0 \mathrm{wt} \%$ of PVA prepared under different cryogenic conditions $(A)$ and with the addition of cryoprotectants prepared in the freezer $\left(-18^{\circ} \mathrm{C}\right)(\mathrm{B})$

It was clear that the freezing rate (Figure 1a) had a significant effect on the transparency of the hydrogels prepared by crosslinking the same solution. The samples frozen in the ice bath appear to be the most transparent. According to the results, highly transparent PVA hydrogels can be created thanks to slow freezing and the associated slow growth of ice crystals. The samples with different concentrations of polyvinyl alcohol prepared in the ice bath and in the freezer $\left(-18^{\circ} \mathrm{C}\right)$ were investigated as well. The results showed that the polymer concentration did not have a significant effect on the final optical properties. 
As mentioned, the kinetics of the ice crystal formation during freezing have a key influence on the optical properties of the resulting PVA hydrogels. Suitable gelation conditions can be therefore ensured by slow gelation at a temperature close to the freezing point of water. However, this procedure is time-consuming and also difficult to automate. Therefore, the effect of the cryoprotectants on the transparency of PVA hydrogels at higher (freezer, $-18{ }^{\circ} \mathrm{C}$ ) than optimal freezing rates was proposed and tested as an alternative approach. Cryoprotectants are substances used to protect biological tissue from freezing damage (i.e. that due to ice formation). The results show (Figure 1b) that the selected cryoprotectants did not significantly affect the optical properties except for urea, which alone affected the optical properties and thus appears to be a potentially interesting component allowing further manipulation of the internal structure of PVA hydrogels.

\subsection{Study of mechanical properties}

Rheology was applied to test the mechanical properties. Various series of PVA hydrogels were prepared for rheological measurements, these samples were measured on the MCR 72 rheometer from Anton Paar. Strain sweep test was chosen as suitable for the description of mechanical properties, this test provides information about the linear viscoelastic region (LVR, it tells us where the moduli are independent on the amplitude of deformation). The data obtained during the measurement were evaluated and processed in the MS Excel software in the form of the dependence of elastic (G') and viscous (G") moduli on the changing amplitude of deformation (Figure 2 shows an example for different cryogenic conditions). The values of phase angles were calculated from viscoelastic moduli. These values provide information about a relative contribution of elastic and viscous types of deformation. Furthermore, the values of the complex modulus as a parameter characterizing the total rigidity of the samples were determined.

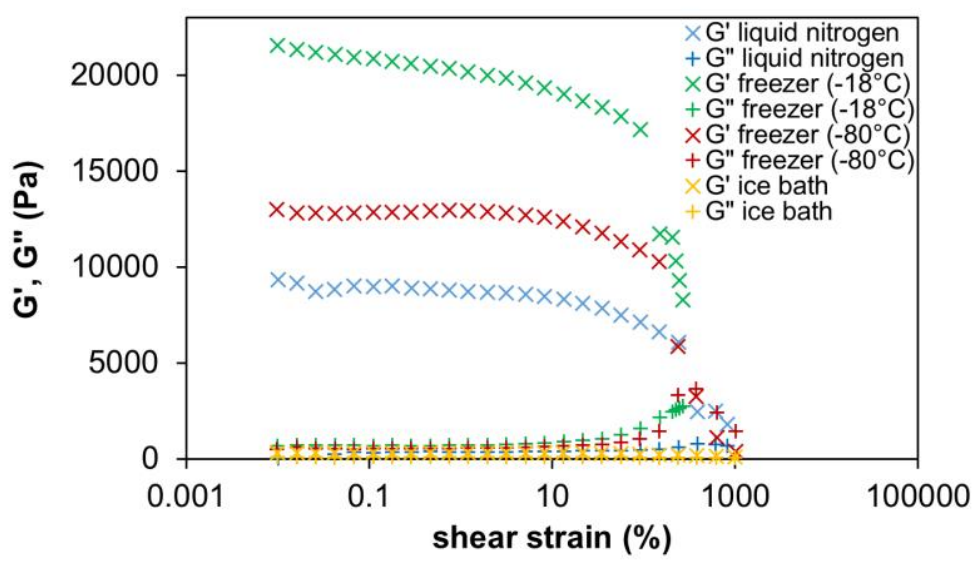

Figure 2 Dependence of elastic and viscous moduli on the changing amplitude of deformation for samples with concentration $10.0 \mathrm{wt} \%$ of polyvinyl alcohol prepared under the different temperature of freezing

The samples prepared in the ice bath were different from the others. The difference in the phase angles (Figure 3a) can be considered as a sign of a difference in the character of the junctions of the hydrogel network. As is known, the rate of freezing of water (depending on the cryogenic conditions) affects the rate of growth of ice crystals, which push the polymer chains at the phase interface. For this reason, in the freezers and the liquid nitrogen bath, contact of multiple chains is achieved in a short time, which creates conditions for multiple physical connections. In the ice bath, due to the relatively high temperature, there is a slow growth of small crystals, which approaches a smaller number of chains and forms fewer junctions, less resistant to deformation. The values of complex modulus (Figure $\mathbf{3 b}$ ) support the conclusions obtained from the comparison of phase angles, which provide information mainly on the quality of the junctions of the hydrogel network and supplement it with information on the density of crosslinking. 
The effect of the addition of cryoprotectants on the mechanical properties was also monitored, but there were no obvious differences between the samples with and without cryoprotectants, respectively.
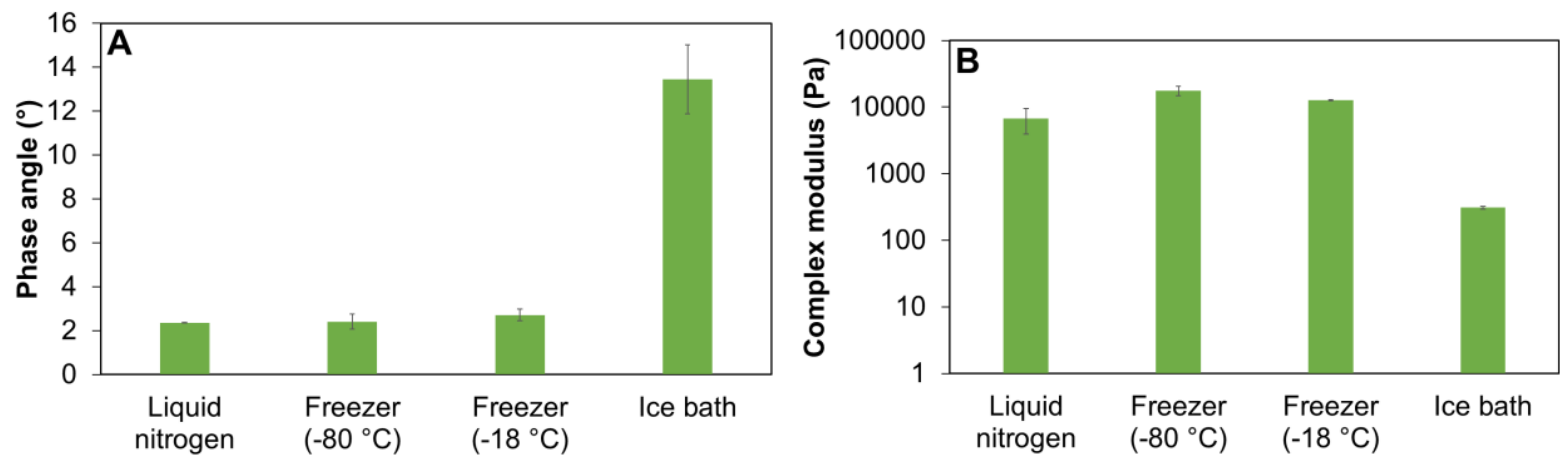

Figure 3 Phase angles (A) and complex moduli (B) for the samples with concentration $10.0 \mathrm{wt} \%$ of polyvinyl alcohol prepared under the different cryogenic conditions

The series of samples with different concentrations of PVA prepared in the freezer $\left(-18{ }^{\circ} \mathrm{C}^{\circ}\right)$ and in the ice bath, respectively, were also compared. The sample with the concentration of $7.5 \mathrm{wt} \%$ prepared in the ice bath behaved viscously. This can be caused by incomplete gelation of the sample, which could be due to the insufficient gelation time. For the other samples, the values of the phase angle in LVR are below the limit of $45^{\circ}$ and these samples therefore already show an elastic character. As the PVA content in the samples increases, the phase angle decreases (Figure 4a). Comparing the values of complex modulus (Figure 4b) again supports the information obtained from the comparison of phase angles. Samples prepared in the freezer have high values of complex modulus. This indicates their high rigidity, which does not differ with increasing PVA concentration. Conversely, the samples prepared in the ice bath show a trend that the rigidity increases with increasing concentration. Thus, as the values of the phase angles decrease the stiffness of the samples increases, which is most likely a sign of an increasing concentration of junctions in the gel network.
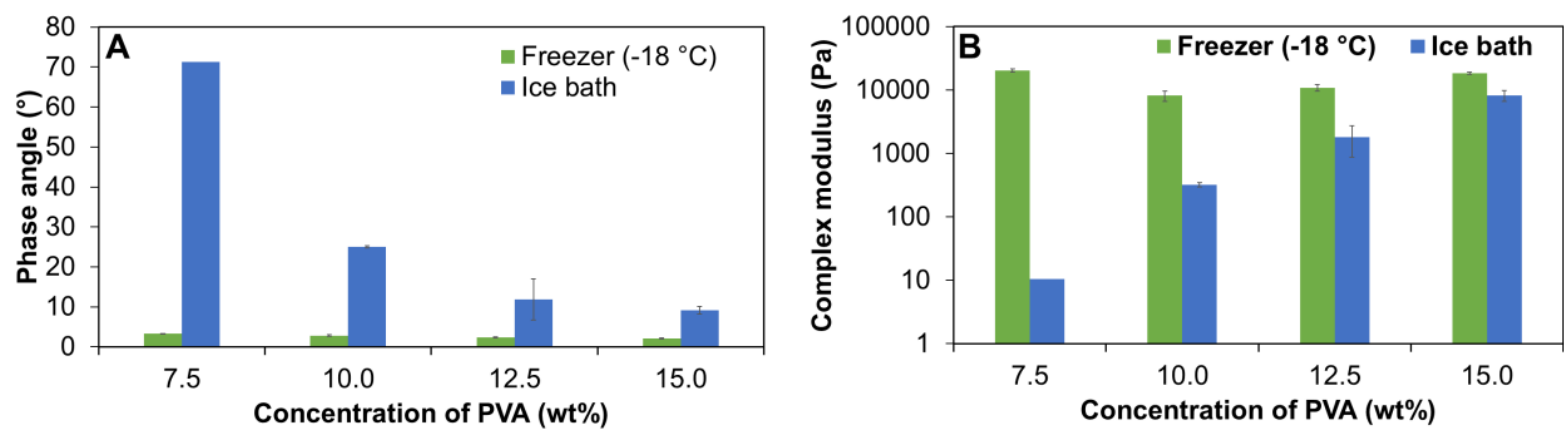

Figure 4 Phase angles (A) and complex moduli (B) for samples with different concentrations of polyvinyl alcohol prepared in the freezer $\left(-18^{\circ} \mathrm{C}\right)$ and in the ice bath

\subsection{Study of microstructure}

Selected hydrogels were analyzed by direct visualization method in their dried form. Scanning electron microscopy was chosen as a suitable method. The purpose was to obtain information about the internal structure of selected hydrogels to support the hypothesis associated with their properties. For this purpose, the selected samples were freeze-dried (after shock-freezing in liquid nitrogen - to preserve the internal structure of the hydrogels without artifacts [8]). Then small parts of the specimens were taken. These specimens were gold coated in a sputtering device and investigated using a scanning electron microscope ZEISS EVO LS 10 with detection of secondary electrons (SE). Hydrogels selected for this analysis were with 


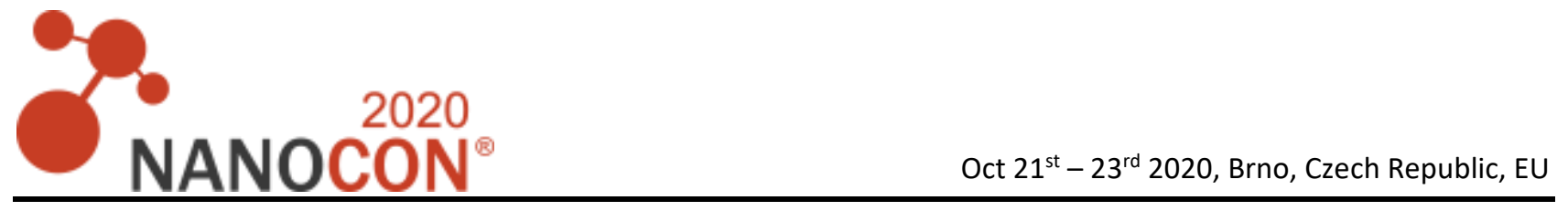

different concentrations of PVA $\left(-18^{\circ} \mathrm{C}\right)$ and samples with 10.0 wt\% of PVA prepared under different temperatures, thus with different freezing rates. A significant part of the samples had a collapsed structure on most of their surface due to the partial recrystallization of water.

A set of samples prepared at different freezing rates was observed first (Figure 5). These samples have a clearly visible porous structure with different pore sizes, which depended on the temperature of freezing and ranged from hundreds of nanometers to several micrometers. For the samples with different concentrations of PVA prepared in the freezer (Figure 6), there is a visible trend in the change of pore size despite the collapsed structure. With increasing concentration of polymer content, the pore sizes decrease. This is due to the higher content of polymer (gelling component), which provides a higher crosslinking density. Hydrogels in the concentration range of 2.5 to $7.5 \mathrm{wt} \%$ of PVA have almost identical porous structure with pore sizes from tens of micrometers (hydrogels with $2.5 \mathrm{wt} . \% \mathrm{PVA}$ ) to hundreds of nanometers (hydrogels with $7.5 \mathrm{wt} . \% \mathrm{PVA}$ ). The sample with $10.0 \mathrm{wt} \%$ of PVA appears as a disordered tangle of fibers, slowly merging into a smooth surface. The remaining two samples with 12.5 and 15.0 wt\% of PVA look completely smooth. It is possible that for these two samples, spots with preparation artifacts were selected for the analysis. Nevertheless, with a higher content of the polymer, it is less likely that the sample will retain its original structure after freeze-drying.

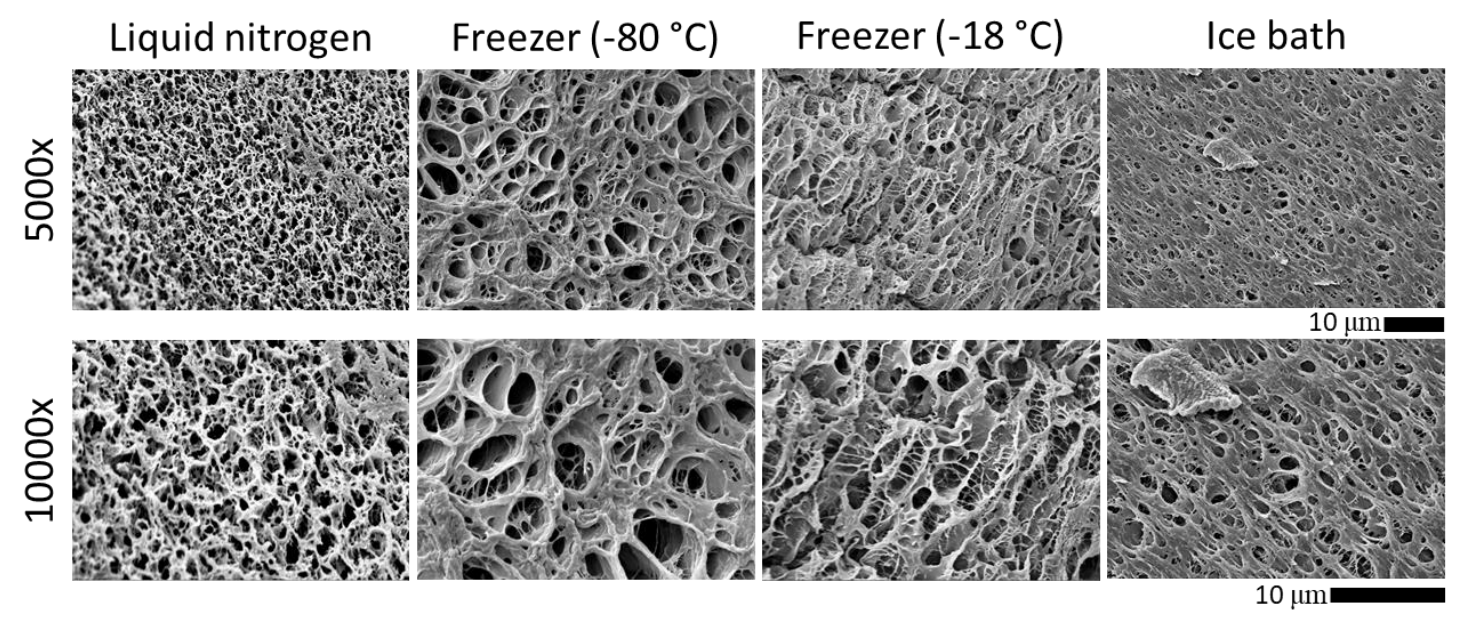

Figure 5 Micrographs of samples with concentration $10.0 \mathrm{wt} \%$ of polyvinyl alcohol prepared under different temperatures of freezing with magnification 5000x and 10000× (SEM ZEISS EVO LS 10)
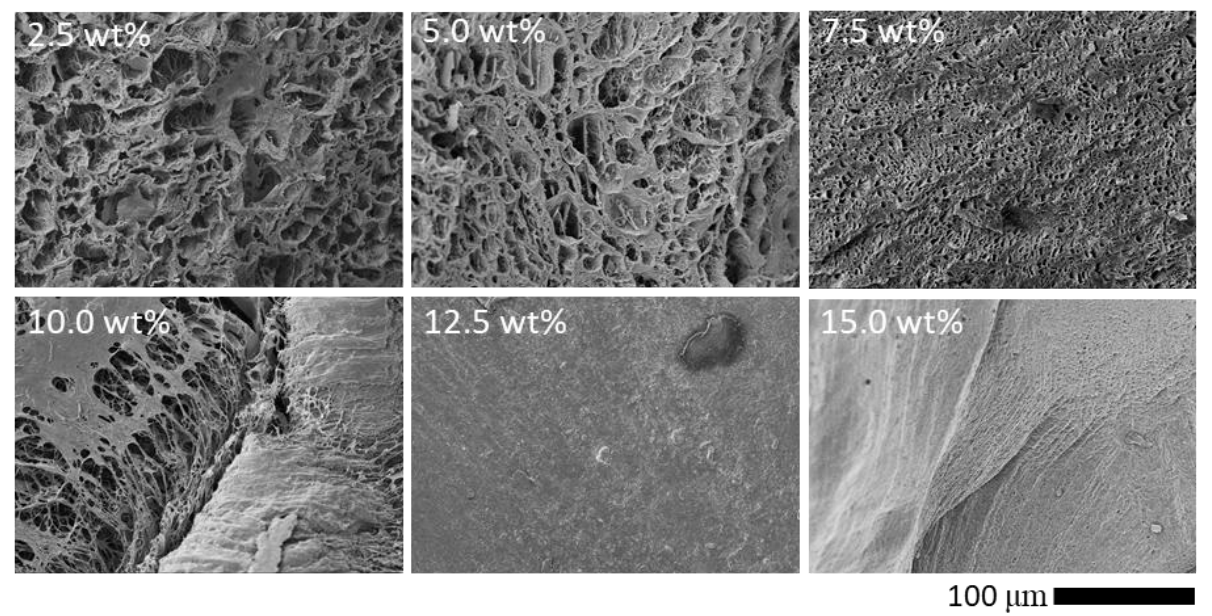

Figure 6 Micrographs of samples prepared in the freezer $\left(-18{ }^{\circ} \mathrm{C}\right)$ with different concentrations of polyvinyl alcohol with magnification 1000× (SEM ZEISS EVO LS 10) 


\section{CONCLUSION}

This work focused on studying properties of physically crosslinked hydrogels with different concentrations of polyvinyl alcohol (2.5 to $15.0 \mathrm{wt} \%)$ prepared via cyclic freezing/thawing under different cryogenic conditions: in the liquid nitrogen $\left(-196{ }^{\circ} \mathrm{C}\right)$, in two different laboratory freezers $\left(-80^{\circ} \mathrm{C}\right.$ and $\left.-18^{\circ} \mathrm{C}\right)$ and in the ice bath. Furthermore, the effects of the addition of several cryoprotectants were evaluated. The optical and mechanical properties of the prepared samples were studied, and the microstructure of the hydrogels was observed by scanning electron microscopy after shock-freezing followed by freeze-drying.

The above results show that the properties of polyvinyl alcohol hydrogels can be varied in many ways. One way to effectively change the structure and properties of hydrogels prepared from polyvinyl alcohol by cyclic freezing/thawing is controlling the freezing rate, which can be affected by adjusting the freezing temperature. By changing the freezing rate, all monitored properties were altered. The cryoprotectants we chose did not sufficiently affect the resulting hydrogels. Therefore, these cryoprotectants are not suitable as additives to alter the properties of polyvinyl alcohol-based hydrogels. The concentration of polyvinyl alcohol in the hydrogel partially changes the properties of the resulting hydrogel. In this case, however, it is necessary to focus on the freezing rate. Because when the sample is frozen in a regular freezer, it is achieved the contact of more chains in a short time independent of the concentration of PVA. However, for slower freezing (the ice bath), due to the relatively low temperature gradient, there is a slow growth of small crystals, which approach a smaller number of chain contacts and form fewer junctions than for rapid freezing and this is the reason why the impact of changing the concentration will be greater for these samples.

\section{ACKNOWLEDGEMENTS}

\section{The research was supported by project COST LD15047.}

\section{REFERENCES}

[1] PEPPAS, N. Hydrogels in pharmaceutical formulations. European Journal of Pharmaceutics and Biopharmaceutics. 2000, vol. 50, no. 1, pp. 27-46.

[2] LI, X., SUN, Q., KAWAZOE, N. CHEN, G. Functional Hydrogels With Tunable Structures and Properties for Tissue Engineering Applications. Frontiers in Chemistry. 2018, vol. 6.

[3] CURVELLO, R., RAGHUWANSHI V.S., GARNIER, G. Engineering nanocellulose hydrogels for biomedical applications. Advances in Colloid and Interface Science. 2019, vol. 267, pp. 47-61.

[4] MEANS, A.K., GRUNLAN, M.A. Modern Strategies To Achieve Tissue-Mimetic, Mechanically Robust Hydrogels. ACS Macro Letters. 2019, vol. 8, no. 6, pp. 705-713.

[5] BUWALDA, S.J., BOERE, K.W.M., DIJKSTRA, P.J., FEIJEN, J., VERMONDEN, T., HENNINK W.E. Hydrogels in a historical perspective: From simple networks to smart materials. Journal of Controlled Release. 2014, vol. 190, pp. 254-273.

[6] BARUI, Ananya. Synthetic polymeric gel. In: Polymeric Gels. Woodhead Publishing, 2018, p. 55-90.

[7] HOLLOWAY, J. L., LOWMAN, A.M., PALMESE, G.R. The role of crystallization and phase separation in the formation of physically cross-linked PVA hydrogels. Soft Matter. 2013, vol. 9, no. 3, pp. 826-833.

[8] RAGHUWANSHI, V.S., GARNIER, G. Characterisation of hydrogels: Linking the nano to the microscale. Advances in Colloid and Interface Science. 2019, vol. 274. 